the worker's performance whilst at work due to ill health (i.e. presenteeism). A number of global measures have been developed to assess presenteeism in clinical studies. However, limited information is available on the correlation between these measures and the construct validity of these measures.

Objectives: To i) determine the correlation between four global measures of presenteeism and ii) to evaluate the construct validity of these measures.

Methods: The main aim of this international observational study (7 countries in Europe and Canada), recruiting patients with IA (RA, PSA or AS) or $\mathrm{OA}$ in paid employment, was to investigate content and construct validity of global presenteeism measures. Patients completed 4 global measures (Work Productivity Scale - Arthritis (WPS-A), Work Productivity and Activity Impairment Questionnaire (WPAI), Work Ability Index (WAI), and both the Quality and Quantity scales of the $Q Q$ questionnaire) (see table legend for descriptions individual scales). Spearman correlations were applied to test the correlation between individual presenteeism scales and to test construct validity with the 11-item Workplace Activity Limitation presenteeism Scale (WALS) and several health related patient reported outcome measures. Interpretation of correlation coefficients: (very) weak ( $\left.r_{\text {range }}=0.0-0.39\right)$, moderate $\left(r_{\text {range }}=0.40-0.59\right)$ to strong $\left(r_{\text {range }}=0.60-1.0\right)$.

Results: 468 patients with a median disease duration of 10 [IQR 5-18] yrs were included; $62 \%$ were female. Median [IQR] presenteeism scores were, respectively: 2 [0-5] for WPS-A, 3 [1-5] for WPAI, 8 [6-9] for WAI, 81 [49-100] QQ-total, with WAI and QQ having reversed scales (Legend table). Correlations between the 4 global measures were moderate to strong, ranging from -0.49 for the correlation between WPS-RA and QQ-Quality to 0.83 between WPAI and WPS-RA. The multiitem presenteeism scale WALS, measuring difficulty at work, strongly correlated with both WPAI $(r=0.65, p<0.05)$ and WPS-RA $(r=0.64, p<0.05)$, both measures capturing the affect and interference of arthritis on work productivity. Moderate correlations were achieved between all six global presenteeism measures and health outcomes ( $r_{\text {range irrespective direction }}$ : $\left|r_{\text {presenteeism-VAS Well-being }}\right|=0.36$ to 0.54 ; $\left|r_{\text {presenteeism-EQ-5D }}\right|=0.37$ to 0.54 ; and $\left|r_{\text {presenteeism-HAQ }}\right|=0.40$ to 0.58$)$.

\begin{tabular}{|c|c|c|c|c|c|c|c|}
\hline & & WPAI & WPS-RA & WAI & $\begin{array}{c}\text { QQ. } \\
\text { Quantity }\end{array}$ & $\begin{array}{c}\text { QQ. } \\
\text { Quality }\end{array}$ & QQ-Total \\
\hline \multirow{6}{*}{$\begin{array}{l}\text { Global presenteeism } \\
\text { scales }\end{array}$} & WPAI & 1.0 & & & & & \\
\hline & WPS-RA & 0.83 & 1.0 & & & & \\
\hline & WAI & -0.65 & -0.62 & 1.0 & & & \\
\hline & QQ-Quantity & -0.58 & -0.53 & 0.60 & 1.0 & & \\
\hline & QQ.Quality & -0.52 & -0.49 & 0.58 & 0.75 & 1.0 & \\
\hline & QQ-Total & -0.60 & -0.56 & 0.63 & 0.95 & 0.88 & 1 \\
\hline $\begin{array}{l}\text { Multi-item } \\
\text { presenteeism scale }\end{array}$ & WALS & 0.65 & 0.64 & -0.55 & -0.50 & -0.49 & -0.54 \\
\hline \multirow{3}{*}{$\begin{array}{l}\text { Healthrelated } \\
\text { patients reported } \\
\text { outcomes }\end{array}$} & VASWell being & 0.54 & 0.51 & -0.43 & -0.39 & -0.36 & -0.42 \\
\hline & EQ-5D & -0.54 & -0.54 & 0.48 & 0.37 & 0.39 & 0.42 \\
\hline & HAQ & 0.57 & 0.58 & -0.52 & -0.40 & -0.41 & -0.45 \\
\hline
\end{tabular}

WPAI ( $0=$ condition no effect on work $-10=$ condition completely prevented work); WPS-IA/OA $(0=$ no interference $10=$ complete interference; WAI ( $0=$ completely unable to work - $10=$ work ability at its best); QQ-Quantity/Quality

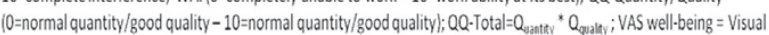
Analogue 5 cale general Well being; EQ-5D=EuroQol 50 measurement; $H A Q=$ health assessment questionnaire.

Conclusions: Global measures of presenteeism show good to moderate construct validity, with the WPAI and WPS-IA/OA showing slightly better construct validity compared to the WAI and $Q Q$. The information obtained in this study will further inform research on instrument use for a standardized approach to estimate presenteeism in future clinical studies.

Acknowledgements: Funding: EULAR, AbbVie, BMS

Disclosure of Interest: None declared

DOI: 10.1136/annrheumdis-2017-eular.5125

\section{THU0690 SERUM AMYLOID A LEVELS AS A POTENTIAL BIOMARKER TO MONITOR PSORIATIC ARTHRITIS PATIENTS ON BIOLOGICS - A RETROSPECTIVE OBSERVATIONAL STUDY}

T. Martins Rocha, R. Fonseca, D. Rosa-Goncalves, F. Aguiar, R. Ferreira, A. Bernardo, M. Bernardes, L. Costa. Rheumatology, Centro Hospitalar de São João, Porto, Portugal

Background: According to previous studies, serum amyloid A (SAA) is involved in the pathophysiology of several conditions including inflammatory arthritis and psoriasis. Recent evidence suggests its valuable role in monitoring disease activity in Rheumatoid Arthritis, but its role is yet to be determined in Psoriatic Arthritis (PsA).

Objectives: To study the association between SAA levels and its variation with other biomarkers and disease activity/functional parameters in a cohort of PsA patients under biologic therapy.

Methods: Observational retrospective study was conducted including PsA patients (according to CASPAR criteria) followed at our Rheumatology department with at least one measurement of SAA levels from January 2015 until December 2016. Demographic and clinical data were obtained by consulting the national database (Reuma.pt). The disease activity/functional scores from at least one visit and corresponding measurements of SAA, ESR and CRP levels were collected. The difference $(\Delta)$ between 2 evaluations separated by a median time of 6 months [6-18] was calculated for all variables. Agreement between dichotomized biomarkers was calculated using kappa coefficients. Correlations were studied using Pearson and Spearman coefficient analysis. Significance level was set as 0.05 .

Results: 53 PsA patients were included. 31 (59\%) patients were females with a mean (SD) age of 50 (11.2) years and a median disease duration of 9 years [1-43]. $28 \%$ had axial involvement, $34 \%$ peripheral involvement and $38 \%$ had both types. All patients were under biologic DMARD. 100 SAA measurements were collected. Median SAA and ESR levels were significantly superior in female patients (23 vs $6 \mathrm{~mm} / 1$ sth and 8.6 vs $4.4 \mathrm{mg} / \mathrm{L}$, respectively, $\mathrm{p}<0.05$ ) and only ESR levels correlated with age $(r=0.20, p=0.05)$. The three biomarkers showed a weak association with serum creatinine levels, with greater correlation for SAA $(r=0.46, p<0.001)$. SAA levels had a stronger correlation with CRP $(r=0.75$, $p<0.001)$ than with ESR levels $(r=0.26, p<0.01)$. SAA and CRP (dichotomized as negative/positive) had a greater level of agreement $(\kappa=0.40)$ compared to ESR ( $\kappa=0.26$ and $\kappa=0.32$, respectively). No significant correlations were found between the biomarkers and the tender/swollen joint count or the pain/global disease activity VAS. SAA levels correlated with ASDAS CRP $(r=0.43, p<0.001)$ and weakly with ASDAS ESR and DAS28 CRP ( $r=0.20$ and $r=0.24$, respectively, $p<0.05)$. Only ESR had a significant weak correlations with BASDAI, MASES and SPARCC scores $(r=0.25, r=0.21, r=0.35 ; p<0.05)$. All the biomarkers had weak correlations with BASFI and HAQ scores. $\triangle$ SAA levels had a weak correlation with $\triangle \mathrm{CRP}(\mathrm{r}=0.32, \mathrm{p}=0.03 ; \mathrm{n}=47)$ and no significant association was found with $\triangle$ ESR. $\triangle$ SAA correlated significantly with $\triangle$ ASDAS CRP and $\triangle B A S M I(r=0.32$, $r=0.39 ; p<0.05$ ).

Conclusions: This study showed that SAA levels and its variation had a significant correlation with CRP levels and its variation, respectively. Significant association with ASDAS CRP variations suggests that serial measurements of SAA may represent an additional marker for monitoring disease activity over time in PSA patients.

References:

[1] Connolly M et al. Arthritis Rheum. (2012);64(4):1035-45.

[2] Hwang et al. Arthritis Res Ther (2016) 18:108.

[3] Morizane S. et al. J Invest Dermatol. (2016) Oct 20. pii: S0022-202X(16)325337.

Disclosure of Interest: None declared

DOI: 10.1136/annrheumdis-2017-eular.5466

\section{THU0691 IS SERUM AMYLOID A PROTEIN AN USEFUL BIOMARKER TO MONITOR TREATMENT WITH ANTI-TUMOUR NECROSIS FACTOR-ALPHA AGENTS IN SPONDYLOARTHRITIS PATIENTS?}

F. Aguiar, D. Rosa-Gonçalves, T. Martins-Rocha, R.M. Ferreira, A. Bernardo, I. Brito, M. Bernardes. Rheumatology, Centro Hospitalar São João, Oporto, Portugal

Background: Quantitating the degree of inflammation has become essential to tailor the treatment strategy of various rheumatic diseases. Traditionally used measures to assess disease activity and treatment response in spondyloarthritis (SpA) are ESR, CRP and Bath Ankylosing Spondylitis Disease Activity Index (BASDAI) and Ankylosing Spondylitis Disease Activity Score (ASDAS). Serum amyloid A (SAA) is an acute-phase reactant predominantly synthesized in the liver by hepatocytes in response to proinflammatory cytokines. Some studies have shown that SAA can be a valuable indicator of disease activity, damage and functional impairment, however it has not been extensively used in clinical practice.

Objectives: To investigate if SAA levels have better correlation with conventional assessments used to monitor anti-tumor necrosis factor $\alpha$ (anti-TNF) treatment in SpA than CRP and ESR

Methods: Prospective study, including SpA patients under anti-TNF $\alpha$ treatment at a Rheumatology Department of a Portuguese Universitary Hospital. The following parameters were collected and registered in two different evaluations 6 months apart from each other: SAA, CRP and ESR levels, BASDAI, ASDASPCR, ASDAS-VS, BASFI, BASMI, swollen and tender joints counts (SJC and TJC), MASES, SPARCC, and patient global assessment (PtGA), physician global assessment (PGA), total back pain (TBP), nocturnal back pain (NBP) measured in a visual analogue scale (VAS). The variation of each parameter was calculated as the difference between the levels registered at each evaluation (baseline and 6 months) and presented as $\Delta$ (parameter). We compared the correlation between $\triangle S A A, \triangle C R P$ and $\triangle E S R$ levels with $\triangle B A S D A I, \triangle A S D A S-P C R, \triangle A S D A S-V S$, $\triangle$ BASFI, $\triangle$ BASMI, $\triangle$ SJC, $\triangle$ TJC, $\triangle$ MASES, $\triangle$ SPARCC, $\triangle$ PtGA-VAS, $\triangle$ PGAVAS, $\triangle T B P-V A S, \triangle N B P-V A S$. The statistical analysis was performed using SPSS 21.0 software, and $p<0.05$ was taken to indicate statistical significance. Correlation was calculated using the Spearman rank correlation $(r)$.

Results: 89 patients were included, $58.4 \%(n=52)$ were male. On baseline the median age was 44.0 years (range 21.0-74.6) and median disease duration was 18.8 years (2-51.6). $\triangle$ SAA was moderately correlated with $\triangle \mathrm{CRP}(r=0.65$, 
$\mathrm{p}<0.001)$ and had lower correlation with $\triangle \mathrm{ESR}(\mathrm{r}=0.28, \mathrm{p}=0.009) . \quad \triangle \mathrm{SAA}$ correlated with $\triangle$ NBP-VAS $(r=0.260, p=0.016)$, but ESR and CRP did not correlate with this parameter. We also found statistically significant correlation between $\triangle$ SAA and $\triangle$ ASDAS-VS $(r=0.257, p=0.017), \triangle$ ASDAS-CRP $(r=0.387$, $p<0.001), \triangle B A S F I(r=0.301, p=0.005) . \triangle C R P$ also showed significant correlation with $\triangle \mathrm{BASF}$, but it was weaker than that observed with $\triangle \mathrm{SAA}(\mathrm{r}=0.230$, $\mathrm{p}=0.033$ ). There was no statistically significant correlation between $\triangle S A A$ levels and $\triangle$ MASES, although $\triangle \mathrm{CRP}$ had a weak correlation $(\mathrm{r}=0.217, \mathrm{p}=0.041)$. There was no significant correlation between neither $\triangle \mathrm{SAA}, \triangle \mathrm{CRP}$ or $\triangle \mathrm{ESR}$ and the following parameters: $\triangle$ TBP-VAS, $\triangle$ PtGA-VAS, $\triangle$ PGA-VAS $\triangle$ BASDAI, $\triangle$ BASMI, $\triangle$ SPARCC, $\triangle T$ TJC or $\triangle$ SJC

Conclusions: This study suggests that SAA can be an useful tool in monitoring treatment with anti-TNF $\alpha$ and that could be introduced in clinical practice. However more studies, with larger sample sizes, should be undertaken to better assess this subject.

Disclosure of Interest: None declared

DOI: 10.1136/annrheumdis-2017-eular.5492

\section{\begin{tabular}{|l|l}
\hline THU0692 THE TURKISH VALIDATION AND RELIABILITY OF \\
\hline
\end{tabular} DISEASE-SPECIFIC, PATIENT REPORTED OUTCOME MEASURE IN RHEUMATOID ARTHRITIS: TR AIMS2-SF}

T. Duruoz ${ }^{1}$, C. Unal Ertekin ${ }^{2}$, C. Sanal Top ${ }^{2} .{ }^{1} P M \& R$ Department, Rheumatology Division; ${ }^{2}$ PM\&R Department, Marmara University, School of Medicine, Istanbul, Turkey

Background: Rheumatoid arthritis (RA) can have a major impact on health related quality of life (HRQoL). The revised AIMS-2 is the main outcome measure that has been used for measuring HRQoL in patients with rheumatic diseases. Guillemin et al. developed a short form of AIMS-2 (AIMS-2 SF) (1) which is more practical and less time consuming compared to AIMS-2.

Objectives: The purpose of this study was to investigate validity and reliability of the Turkish version of AIMS2-SF (TR AIMS-2 SF).

Methods: Turkish AIMS2-SF was developed after translation and back-translation method. Culturally adapted version preserved 5 component-structure (upper limb function, lower limb function, affect, pain and social interaction) with 26 items according to the original article (1). Subjects fulfilling ACR/EULAR 2010 classification criteria for RA were consecutively enrolled into the study. Patients with malignancy, fibromyalgia syndrome and other systemic inflammatory diseases were excluded. Demographic data, the body mass index (BMI), severity of pain (VAS), disease duration (month) and other clinical features was evaluated. Reliability was investigated with test-retest reliability (intraclass correlation coefficient-ICC) and internal consistency (Cronbach's alpha). Spearman's rank correlation coefficient was used to evaluate the relation between quantitative parameters and the validity. Construct validity was assessed by the correlation of TR AIMS2-SF with other clinical parameters (age, disease duration, VAS pain, DAS-28) and functional parameters such as Nottingham Health Profile (NHP), Health Assessment Questionnaire (HAQ), Beck Depression Inventory (BDI), Duruoz Hand Index (DHI). Statistical analyses were performed with SPSS version 20 and a value of $p<0.05$ was considered as statistically significant.

Results: Sixty patients $(6$ males) were recruited into the study. The mean \pm standart deviation (SD) of age (years) and disease duration (months) were $51.8 \pm 12.5$ and $71.4 \pm 69.3$, respectively. Mean scores of TR AIMS2-SF were; upper limb function $7.3 \pm 6.9$, lower limb function $7.7 \pm 4.7$, affect $7.4 \pm 3.2$, pain $6 \pm 3.1$, social interaction $4 \pm 2.3$ and total score $35.5 \pm 16.6$. The floor and ceiling effects of TR AIMS2-SF were 1.6 and 3.3, respectively. Both Cronbach's alpha and ICC were 0.83 indicating good reliability. There was significant correlation (rho, $\mathrm{p}$ value) with parameters that were directly related to $\mathrm{HRQ}$ oL which were NHP subscales (energy level: 0.46 , pain: 0.63 , emotional reaction: 0.55 , sleep 0.33 , social interaction: 0.60 , physical activity: $0.63 ; p<0.0001), \mathrm{HAQ}(0.70, p<0.0001)$, BDI $(0.54, p<0.0001)$, DHI $(0.60, p<0.0001)$. Poor or not significant correlation was found with parameters that were not directly related to HRQoL such as age $(-0.004, p=0.97)$, disease duration $(0.21, p=0.09)$, vas pain $(0.37, p<0.05)$; on the other hand, disease activity (DAS-28) correlated moderate $(0.49, \mathrm{p}<0.0001)$.

Conclusions: Turkish version of AIMS2-SF is a reliable and valid tool that can be used to evaluate the quality of life in RA. This is a feasible measure that can be used in daily practice easily.

References:

[1] Guillemin F, Coste J, Pouchot $\mathrm{J}$ et al. The AIMS2-SF: a short form of the Arthritis Impact Measurement Scales.

[2] French Quality of Life in Rheumatology Group. Arthritis Rheum 1997, 40:1267-74.

Disclosure of Interest: None declared

DOI: 10.1136/annrheumdis-2017-eular.6351

\section{THU0693 FEASIBILITY AND RELIABILITY OF THE SPONDYLOARTHRITIS RESEARCH CONSORTIUM OF CANADA SACROILIAC JOINT INFLAMMATION SCORE FOR CHILDREN WITH SPONDYLOARTHRITIS}

N.A. Chauvin ${ }^{1}$, W.P. Maksymowych ${ }^{2}$, R.G. Lambert ${ }^{3}$, J.L. Jaremko ${ }^{3}$, D.M. Biko ${ }^{1}$, J. Paschke ${ }^{4}$, T.G. Brandon ${ }^{5}$, P.F. Weiss ${ }^{5} .{ }^{1}$ Radiology, Children's
Hospital of Philadelphia, Philadelphia, United States: ${ }^{2}$ Rheumatology;

${ }^{3}$ Radiology, University of Alberta; ${ }^{4}$ Care Arthritis, Alberta, Canada;

${ }^{5}$ Rheumatology, Children's Hospital of Philadelphia, Philadelphia, United States

Background: Clinical trials in children with pediatric spondyloarthritis and axial disease are lacking. In order to assess the effectiveness of medications we need an objective measure to quantify severity of inflammation in the pediatric sacroiliac joint.

Objectives: We evaluated the reliability and construct validity of the Spondyloarthritis Research Consortium of Canada (SPARCC) sacroiliac joint inflammation score (SIS) in children with suspected or confirmed juvenile spondyloarthritis (SpA).

Methods: The SIS divides the joint into quadrants and scores the presence, depth, and intensity of bone marrow edema (BME) on short tau inversion recovery (STIR) magnetic resonance imaging (MRI). Six consecutive semicoronal slices through the cartilaginous portion of the joint are scored for BME (total score $0-72$ ). We developed a pediatric training module that included scoring instructions and examples of bright subchondral signal on STIR scans easily confused with inflammation (Figure). After reviewing the module, 6 readers ( 1 adult and 3 pediatric radiologists, 1 adult rheumatologist, and 1 pediatric rheumatologist), blinded to clinical details except age, scored 30 studies that included semicoronal T1-weighted and STIR sequences. Pain was recorded on a visual analogue scale $(0-10)$. Disease activity was evaluated using the juvenile SpA disease activity (JSPADA) index (range 0-8). Inter-observer reliability was assessed using intraclass correlation (ICC). Correlation (convergent validity) of the mean SPARCC SIS developers' score with disease activity was tested using Spearman correlation. Discrimination was tested by comparing the mean SPARCC SIS developers' score between children with and without inflammatory back pain using the Mann-Whitney test.

Results: The SIS had face validity and was feasible to score in the 30 pediatric cases. $21(70 \%)$ were male. Median age at the time of imaging was 15.5 years (IQR: 12.7-16.8). Median pain score and JSpADA index were 2 (IQR 0.5-6) and 2 (IQR: $0.5-3$ ), respectively. Of the 180 scores submitted by 6 readers, 140 $(78 \%)$ of the studies had a SIS $\geq 2$. Median SIS was 14 (IQR: 3-29). ICCs for all readers $(\mathrm{N}=6)$, SPARCC developers $(\mathrm{N}=2)$, rheumatologists $(\mathrm{N}=2)$, and pediatric radiologists $(\mathrm{N}=3)$ were $0.63(95 \% \mathrm{Cl}: 0.45-0.78), 0.89(95 \% \mathrm{Cl}: 0.75-0.95)$, 0.43 (95\% Cl: $-0.02-0.71)$, and 0.85 (95\% Cl: $0.72-0.92)$, respectively. SIS had low correlation with disease activity as measured by the JSpADA ( $r=-0.08)$ and C-reactive protein $(r=0.14)$. SIS score did not discriminate between those with and without inflammatory back pain $(\mathrm{p}=0.16)$.
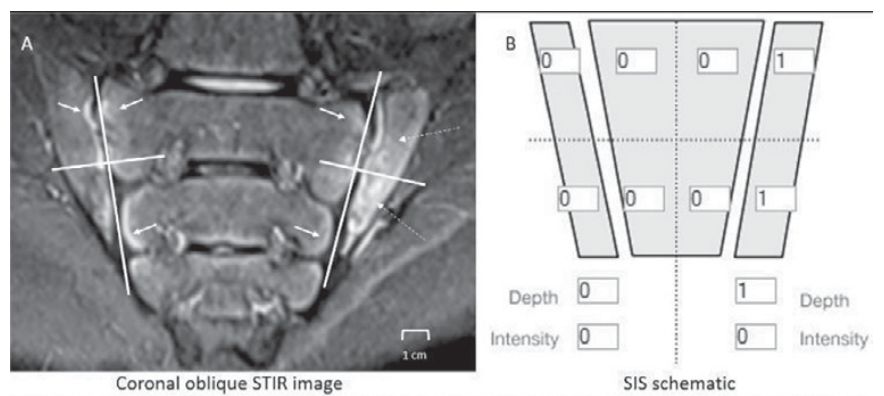

Figure 1. Sample slice and scoring methods for sacroiliac joint inflammation score (SIS). A Coronal oblique STIR image of the sacroiliac joints shows each joint divided into 4 quadrants. There is normal bright subchondral signal along both sides of the right sacroiliac joint and along the periarticular region of the left sacrum (solid arrows) in this skeletally immature patient. On the left, there is abnorma signal consistent with bone marrow edema within periarticular region of the left lilum in both the upper and lower quadrants (dashed arrows). B. Scoring schematic demonstrates a "1" within the left iliac bon in both the upper and lower quadrants representing bone marrow edema. An additional score of " 1 " is given as the depth of the bone marrow edema extends $>1 \mathrm{~cm}$ from the articular surface. The patien received a total score of " 3 " for this image slice.

Conclusions: The SIS was feasible to score and had near excellent reliability, even with limited calibration. SIS did not have convergent validity with clinical measures of disease activity, highlighting that imaging and clinical evaluations provide complimentary but non-overlapping information. Responsiveness of the SIS should be evaluated in a prospective cohort of children who start biologic therapy.

Disclosure of Interest: None declared

DOI: 10.1136/annrheumdis-2017-eular.6328

\section{THU0694 EFFECTS OF ANTI-TNF THERAPY ON SOLUBILE UROKINASE PLASMINOGEN ACTIVATOR RECEPTOR (SUPAR) LEVELS IN ARTHRITIS}

A. Pusztai, E. Végh, Á. Horváth, A. Hamar, S. Szántó, Z. Szekanecz, G. Szücs. Department of Rheumatology, University of Debrecen, Faculty of Medicine, Debrecen, Hungary

Background: The urokinase plasminogen activator receptor (UPAR) is expressed mainly on immune cells, smooth muscle cells and endothelial cells, favoring extracellular matrix degradation, cell adhesion, cell proliferation and regulates cell migration. The suPAR is the soluble form of the cell membrane-bound protein 